\title{
NON-COMMUTATIVE SEPARABILITY AND GROUP ACTIONS
}

\author{
RicARDo AlFARO* \\ Dedicated to the memory of Pere Menal
}

\begin{abstract}
We give conditions for the skew group ring $S * C$ to be strongly separable and $H$-separable over the ring $S$. In particular we show that the $H$-separability is equivalent to $S$ being central Galois extension. We also look into the $H$-separability of the ring $S$ over the fixed subring $R$ under a faithful action of a group $G$. We show that such a chain: $S * G H$-separable over $S$ and $S H$-separable over $R$ cannot occur, and that the centralizer of $R$ in $S$ is an Azumaya algebra in the presence of a central clement of trace one.
\end{abstract}

In [A] we introduced the concept of subring-Galois extensions as a generalization of central Galois extensions and give a generalization of the correspondence theorem given by DeMeyer in [D] and Szeto in [SM]. Similar correspondence theorems were given by Sugano in $[\mathbf{S}]$ using $H-$ separability. Separability for non-commutative rings was introduced by Hirata, and the notions of $H$-separability and "strong" separability were introduced by Hirata in [HI] and MacMahon and Mewborn in [MM] respectively. Strong separability is a weaker notion than $H$-separability, but both are special cases of the general notion of separability of ring extensions.

In the case of group actions we present here conditions for strong and $H$-separability of skew group rings and in particular we show that the skew group ring $S * G$ is $H$-separable over $S$ if and only if $S$ is a central Galois extension. Furthermore, in this case $S * G$ is a $Z(S)$-Galois

*Partially supported by a grant from the Faculty Development Fund of the University of Michigan-Flint and a fellowship from the Centre de Recerca Matematica, Barcelona, Spain. 
extension (in the terminology of $[\mathbf{A}]$ ), allowing us to cxpress $S=Z(S) R$ and $S * G=Z(S) I$ where $I$ is the algebra of $G$-central functions. We then study the separability of $C_{S}(R)$ over its fixed subring and give conditions for $S$ to be $C_{S}(R)$-Galois.

All rings here are associative and have a unity element $1 . Z(R)$ will denote the center of a ring $R$, and $C_{A}(B)$ will denote the "centralizer

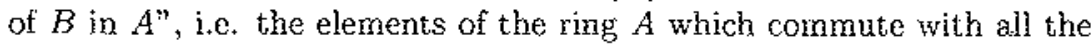
elements of the subring $B$ of $A$.

\section{Definitions and Notations}

Let $B$ be a subring of a ring $A$ with 1 .

The extension $B \subset A$ is called separable (or $A$ is separable over $B$ ) if any of the following equivalent conditions is satisfied:

1) The multiplication map $\mu: A \otimes_{B} A \rightarrow A$ splits as an $(A-A)$ bimodule map.

2) There exists an element $e \in A \otimes_{B} A$ (called a separability element), such that $\alpha e=e \alpha$ for all $\alpha \in A$ and $\mu(e)=1$.

The ring $A$ is said to be strongly separable over $B$ if $A \otimes_{B} A \cong K \oplus L$ as $(A-A)$-bimodules, where $\operatorname{Hom}_{A, A}(K, A)=0$ and $L \Theta H \cong A^{n}$ for some $(A-A)$-bimodules $K, L, H$ and some positive integer $n$. In case $K=0$ we say that $A$ is $H$-separable over $B$. Strongly separable extensions are separable but the converse is false, see [MM].

There is an equivalent definition for this kinds of separability in terms of the natural $(A-A)$-bimodule map $\varphi: A \otimes_{B} A \rightarrow \operatorname{Hom}\left(\Delta_{c}, A_{c}\right)$ where $\varphi(a \otimes b)(x)=a x b, C$ is the center of $A$ and $\Delta$ is the centralizer of $B$ in $A, C_{A}(B)$. The ring $A$ is strongly separable over $B$ if and only if $\Delta_{C}$. is finitely generated projective $C$-module and $\varphi$ is an split epimorphism. Similarly, $A$ is $H$-separable over $B$ if and only if $\Delta_{c}$ is finitely generated projective $C$-module and $\varphi$ is an isomorphism. For details see [HM] and [MM].

Now let's consider group actions. Let $S$ be a ring with 1 , let $G$ be a finite group acting faithfully as automorphisms of $S$ and let $R=S^{G}$ be the fixed ring under $G$. Writing $g(r)={ }^{g} r$, the skew group ring $S * G$ is the free left $S$-module with basis the elements of $G$ and multiplication given by the rule $g s={ }^{g} s g$ for all $s \in S$ and $g \in G$. Denote by $\pi$ the element $\sum_{g \in G} g \in S * G$. The action of $G$ on $S$ is said to be $G$ Galois if $S$ is finitely generated projective right $R$-module and the natural map $\phi: S * G \rightarrow \operatorname{Fnd}_{R} S$ given by $\phi(r g)(x)=r\left({ }^{g} x\right)$ is a ring isomorphism; or equivalently, there exist clements $a_{i}, b_{i}$ (called a $G$-Galois basis) such 
that $\sum_{i} a_{i}{ }^{g} b_{i}=1$ if $g=1$ and the sum is 0 if $g \neq 1$ (i.e., $S \pi S=S * G$ ). The "trace map", $t r: S \rightarrow R$ is given by $\operatorname{tr}(x)=\sum_{g \in G}{ }^{9} x$ which is an $(R-R)$-bimodule homomorphism.

Let $T$ be a $G$-stable subring of $S$ (that is ${ }^{g} t \in T$ for all $t \in T, g \in G$ ), we say that $S$ is a $T$-Galois extension of $R$ if the action of $G$ on $T$ is $G$-Galois. For details and properties, see $[\mathbf{A}]$. If $X$ is a subset of $S$, let $I(X)=\left\{g \in G /{ }^{g} x=x \quad \forall x \in X\right\}$ be the "inertia group" of $X,(I(X)$ is always a subgroup of $G$ ).

\section{Separability and skew group rings}

In [MS, theorems 2.2 and 2.3] it is shown that if $S$ is a simple ring, $G$ a finite outer group of automorphisms of $S$ and $F=I(Z(S))$, then $S * G$ is $H$-separable over $S * F$ and $S * G$ is $H$-separable over $S$ if and only if $F$ is trivial. But in this case $S * G$ is simple and hence the action of $G$ on $S$ is $G$-Galois. We'll give a general result relating $G$-Galois actions with strong and $H$-separability.

Let $D=C_{S * G}(S)$ and $C=Z(S * G)$. The action of $G$ on $S$ induces a faithful action of $G$ on $S * G$ via conjugation, ${ }^{g} \alpha=g \alpha g^{-1}$ for $\alpha \in S * G$; and $G$ also acts on $D$. Let $M$ be the inertia group of $D$, thus $G / M$ acts faithfully on $D$ by ${ }^{\bar{h}} \alpha={ }^{g} \alpha$ for any $g \in \bar{h}$.

Lemma 2.1. $D^{G}=D^{G / M}=C$.

Proof: The first equality is obvious since $M$ is the inertia group of $D$. Now let $\alpha \in D^{G}$, then $\alpha g=g \alpha \quad \forall g \in G$ and by definition of $D$, $s \alpha=\alpha s \quad \forall s \in S$; hence $\alpha \in C$. Conversely, if $\alpha \in C, \alpha g=g \alpha \quad \forall g \in G$ and hence $\alpha \in D^{G}$, (is clear that $C \subseteq D$ ).

Theorem 2.2. Let $M$ be the inertio group of $D=C_{S * G}(S)$ and let $C$ be the center of $S * G$. Assumme there is a central element $w$ in $S$ with $\operatorname{tr}_{M}(w)=1$. If $D$ is $G / M$-Galois over $C$, then $S * G$ is strongly separable over $S$.

Proof: Let $\varphi: S * G \otimes_{S} S * G \rightarrow \operatorname{Hom}\left(D_{C}, S * G_{C}\right)$ be the natural $\left(S * G-S * G\right.$ )-bimodule map, and let $\left\{a_{i}, b_{i}\right\}$ be a $G / M$-Galois basis for $D$ over $C$; then define the maps $f_{i}$ by $f_{i}(x)=\operatorname{tr}_{C / M}\left(b_{i} x\right)$, thus $f_{i} \in$ Hom $\left(D_{C}, C_{C}\right)$ and $\left\{a_{i}, f_{i}\right\}$ form a dual projective basis for $D$ over $C$.

First we show that $\left\{f_{i}\right\}$ is a basis for $\operatorname{Hom}\left(D_{C}, S * G_{C}\right)$ as $(S * G-S * G)$-bimodule. For, let $\alpha \in D, f \in \operatorname{Hom}\left(D_{C}, S * G_{C}\right)$, 
then $f(\alpha)=f\left(\sum_{i} a_{i} f_{i}(\alpha)\right)=\sum_{i} f\left(a_{i}\right) f_{i}(\alpha)=\sum_{i} f_{i}(\alpha) f\left(a_{i}\right)$; thus $f=\sum f\left(a_{i}\right) f_{i}=\sum^{i} f_{i} f\left(a_{i}\right)$. Now we prove that $\varphi$ is an epimorphism. Note that $\varphi\left(g \otimes g^{-1}\right)(\alpha)=g \alpha g^{-1}$, thus $\varphi\left(g \otimes g^{-1}\right)$ acts as $\bar{g} \in G / M$ on $D$ and $\varphi\left(g \otimes g^{-1}\right)=\varphi\left(h \otimes h^{-1}\right)$ whenever $\bar{g}=\bar{h}$ in $G / M(*)$. Choose $\left\{h_{1}, \ldots, h_{p}\right\}$ a transversal of $M$ in $G$, then

$$
\begin{aligned}
f_{j}(x)=\operatorname{tr}_{G / M}\left(b_{j} x\right) & =\sum_{h_{i}}^{h_{2}}\left(b_{j} x\right)=\sum_{h_{i}}^{h_{2}} b_{j}^{h_{2}} x \\
& =\sum_{i}^{h_{i}} b_{j} \varphi\left(h_{i} \otimes h_{i}^{-1}\right)(x)=\sum_{i} \varphi\left(h_{i} b_{j} \otimes h_{i}^{-1}\right)(x) .
\end{aligned}
$$

Therefore $f_{j} \in \operatorname{Im}(\varphi)$ and hence $\varphi$ is epic. Notice that the expression of $f_{j}$ above is independent of the choice of the transversal of $M$ in $G$ by $(*)$. It is only left to show that $\varphi$ splits as $(S * G-S * G)$-bimodule homomorphism. Let $M$ be given by the set $\left\{m_{1}, \ldots, m_{q}\right\}$ and let $l_{k}=$ $\sum_{i, j} h_{i} m_{j} w b_{k} \otimes\left(h_{i} m_{j}\right)^{-1} \in S * G \otimes_{S} S * G$. Then

$$
\begin{aligned}
& \varphi\left(l_{k}\right)=\sum_{i, j}{ }^{h_{i} m_{j}} w^{\overline{h_{i}} \overline{m_{j}}} b_{k} \varphi\left(h_{i} m_{j} \otimes\left(h_{i} m_{j}\right)^{-1}\right) \text { and by }(*) \\
= & \sum_{i, j}{ }_{h_{i} m_{j}} w^{\overline{h_{i}}} b_{k} \varphi\left(h_{i} \otimes h_{i}^{-1}\right)=\sum_{i}\left(\sum_{j}^{h_{i} m_{j}} w\right) \varphi\left(h_{i} b_{k} \otimes h_{i}^{-1}\right)=f_{k} .
\end{aligned}
$$

Hence we may define the map $\psi: \operatorname{Hom}\left(D_{C}, S * G_{C}\right) \rightarrow S * G \otimes_{S} S * G$ by linearity with $\psi\left(f_{k}\right)=l_{k}$. To show that $\psi$ is an $(S * G-S * G)$-bimodule map, we need to show $\alpha l_{k}=l_{k} \alpha$ for all $\alpha \in S * G$. Let $r \in S$, since $b_{k} \in D$ and $w$ is central in $S$ we have:

$$
\begin{aligned}
r l_{k} & =\sum_{i, j} r\left(h_{i} m_{j}\right) w b_{k} \otimes\left(h_{i} m_{j}\right)^{-1} \\
& =\sum_{i, j}\left(h_{i} m_{j}\right)^{\left(h_{i} m_{j}\right)^{-1}} r w b_{k} \otimes\left(h_{i} m_{j}\right)^{-1} \\
& =\sum_{i, j} h_{i} m_{j} w b_{k} \otimes{ }^{\left(h_{i} m_{j}\right)^{-1}} r\left(h_{i} m_{j}\right)^{-1} \\
& =\sum_{i, j} h_{i} m_{j} w b_{k} \otimes\left(h_{i} m_{j}\right)^{-1} r=l_{k} r
\end{aligned}
$$

and if $g \in G$, we have:

$$
g l_{k}=\sum_{i, j} g h_{i} m_{j} w b_{k} \otimes\left(h_{i} m_{j}\right)^{-1}=\sum_{i, j}\left(g h_{2}\right) m_{j} w b_{k} \otimes\left(\left(g h_{i}\right) m_{j}\right)^{-1} g,
$$


but $\left\{g h_{i}\right\}$ is another transversal of $M$ in $G$, hence by $(*) g l_{k}=l_{k} g$ and therefore $\psi$ is an $(S * G-S * G)$-bimodule map. We then have

$$
\begin{aligned}
\varphi(\psi(f)) & =\varphi\left(\psi\left(\sum_{k} f\left(a_{k}\right) f_{k}\right)\right)=\varphi\left(\sum_{k} f\left(a_{k}\right) l_{k}\right) \\
& =\sum_{k} f\left(a_{k}\right) \varphi\left(l_{k}\right)=\sum_{k} f\left(a_{k}\right) f_{k}=f .
\end{aligned}
$$

and so $\psi$ splits $\varphi$.

Now we want to show an equivalent condition for the skew group ring $S * G$ to be $I I$-separable over $S$. We start by giving some notation and some neccesary conditions assuming all the notation as in thoorcm 2.2 .

For every $g \in G$ define $\phi_{g}=\left\{r \in S / r^{g}=s r \quad \forall s \in S\right\}$. If $\phi_{g} \neq 0 \quad g$ is said to be $\omega$-inner, and if $\phi_{g}=0$ for every $g \neq I \quad G$ is said to be $\omega$-outer. It is not difficult to see that $D=\sum_{g \in G} \phi_{g} g$.

For the proof of the main theorem we will need a result that appears in $[\mathbf{A}]$, and we reproduce here for completeness.

Proposition 2.3. ([A, prop. 3.3]) Assume $S * G$ is $H$-separuble over $S$. Then $G$ is $\omega$-outer and $D=Z(S)$.

Proof: Since $S * G \cong \sum_{g \in G}^{\oplus}(S \otimes g)$ as $S$-S-bimodules, $C_{s m G}(D)=S$ by [S, proposition 1.3]. Hence $Z(D)=C_{s . G}(D) \cap D \subseteq S$ and therefore $C \subseteq Z(D) \subseteq Z(S)$. Now let $r_{g} \in \phi_{g}$, so $x=r_{g} g \in D$, and hence $\operatorname{tr}_{G / M}(x)=\sum_{\bar{h} \in G / M} h r_{g} g h^{-1}=\sum_{\bar{h} \in G / M} h_{r_{g}} h g h^{-1} \in C \subseteq S$. Thus ${ }^{h_{r_{g}}}=0$ if $h g h^{-1} \neq 1$, this is if $g \neq 1$ and so $r_{g}=0$ if $g \neq 1$. Therefore $\phi_{g}=0$ if $g \neq 1$, and so $G$ is $\omega$-outer. By the comment above $D=\phi_{1} \cdot 1$, so $D=Z(S)$.

Theorem 2.4. Let $D, M, C, S, G$ and $w$ as in theorem 2.2. $D$ is $G$ Galois over $C$ and $M$ is trivial if and only if $S * G$ is $H$-separable over $S$.

Proof: $\Leftrightarrow$ Assume the same notation as in the proof of theorem 2.2; so now we have $l_{k}=\sum_{i, j} h_{i} w b_{k} \otimes\left(h_{i}\right)^{-1}=\sum_{i} h_{i} b_{k} \otimes h_{i}^{-1}$, and hence

$$
\begin{aligned}
\psi \cdot \varphi(1 \otimes 1) & =\sum_{k} \varphi(1 \otimes 1)\left(a_{k}\right) l_{k}=\sum_{k} a_{k} \sum_{i} h_{i} b_{k} \otimes h_{i}^{-1} \\
& =\sum_{i}\left(\sum_{k} a_{k}{ }^{h_{2}} b_{k}\right) h_{i} \otimes h_{i}^{-1}=1 \otimes 1 .
\end{aligned}
$$


Thus $\psi \cdot \varphi=\mathrm{id}_{S * G \otimes S} S * G$ and $\varphi$ is an isomorphism.

$(\Leftarrow)$ Assume $m \in M$ and $\alpha \in D$, then $\varphi\left(m \otimes m^{-1}\right)(\alpha)=m \alpha m^{-1}=$ $\alpha=\varphi(1 \otimes 1)(\alpha)$, but $\varphi$ is an isomorphism, hence $M=1$. Now we will show $D$ is $G$-Galois over $C$. By proposition $2.3 D$ is commutative, and by $[\mathbf{S}$, proposition 1.3 $D$ is a separable $C$-algebra. Assume that there exists a non zero idempotent $e \in D$ and a pair $h \neq g \in G$ such that ${ }^{g} x e={ }^{h} x e$ for all $x \in D$. If we let $e^{\prime}=g^{-1} e$, we have $e^{f} \neq 0$ and $x e^{\prime}=g^{-1} h x e^{\prime}=e^{\prime} g^{-1} h x$. But $G$ is wwouter, hence $g^{-1} h=1$, thus $g=h$, a contradiction. Therefore $D$ is $G-$ Galois over $C$ by [DI, proposition III. 1.2].

If $S$ is a simple ring and $G$ is outer, then $Z(S)$ is a ficld, and hence $G / M$ is $G / M$-Galois over $Z(S)$ where $M=I(Z(S)$ ). Therefore applying the previous theorems we obtain an improvement of [MS, Theorem 2.3 and Theorem $2.2, \mathrm{ii})]$ :

Corollary 2.5. Let $S$ be a simple ring and $G$ be outer.

i) If $\exists w \in Z(S)$ such that $\operatorname{tr}_{M}(w)=1$, then $S * G$ is strongly separable over $S$.

ii) $S * G$ is $H$-separable over $S$ if and only if $M=1$.

We can see now a relationship between $H$-separability and $T$-Galois extensions in the following corollaries:

Corollary 2.6. $S * G$ is $H$-separable over $S$ if and only if $S$ is a central Galois extension of $R$.

Proof: $(\Leftarrow) \exists a_{i}, b_{i} \in Z(S)$ such that $\sum a_{i} \pi_{G} b_{i}=1$, but $Z(S) \subseteq$ $C_{S * G}(S)=D$ and $D$ is $G$-invariant, hence $D$ is $G$-Galois over $D^{G}=C$ and by theorem $2.4 S * G$ is $H$-separable over $S$.

$(\Rightarrow)$ Obvious from the theorem 2.4 and proposition 2.3 .

The case of commutative rings is now determined:

Corollary 2.7. Let $S$ be a commutative ring. $S * G$ is $H$-separable over $S$ if and only if $S$ is $G-$ Galois over $R$.

Consider again the action of $G$ on $S * G$ by conjugation. It follows that the centralizer of $G$ in $S * G$ is precisely equal to the fixed ring $(S * G)^{G}=I$, which in the language of $C^{*}$ algebras is called the algebra of $G$-central functions, (see [OP]). Hence we obtain:

Proposition 2.8. Let $S * G$ be $H$-separable over $S$. Then $S * G$ is a $Z(S)$-Galois extension of $I$ and therefore $S * G=Z(S) I$. 


\section{3. $H$-separability and fixed ring}

Now we study some neccesary conditions for the ring $S$ to be $H-$ separable over the fixed ring $R$. The centralizer of $R$ in $S$ will be denoted by $E$ and all the notation from Section 2 will be assumed.

Let $X$ be a $G$ invariant subset of $S$. It can be easily seen that $C_{S}(X)$ is a $G$-invariant subring of $S$ and thus $G$ acts on it. Furthermore we have that $\left(C_{S}(X)\right)^{G}=C_{R}(X)$. Hence, if we take $X=R$ we get the following relation: $E^{G}=Z(R) \subseteq Z(E)$. On the other hand it is obvious that $Z(S) \subseteq Z(E)$.

Proposition 3.1. Let $S$ be $H$-separable over $R$. Then:

1) $G$ is $\omega$-inner.

2) $R=C_{S}(E)$

3) $E^{G}=Z(R)=Z(E)$

Proof: 1) Recall that $\phi_{g}=\left\{r \in S / r^{9} s=s r \quad \forall s \in S\right\}$. Consider the $(S-S)$-bimodule $S g$. Then $E_{g}=C_{S g}(R)$ and $\phi_{g} g=C_{S_{g}}(S)$, therefore we get $E g=E \otimes_{Z(S)} \phi_{g} g$ and hence $\phi_{g} \neq 0$.

2) It is clear that $R \subseteq C_{S}(E)$. Now, let $r \in C_{S}(E)$ and let $g \in G$. We can see $g$ as an clement of $\operatorname{Hom}_{R-R}(S, S)$ which is isomorphic to $E \otimes_{Z(S)} E$ by [H2, proposition 4.7]. Thus there exists elements $d_{i}, e_{i} \in E$ such that ${ }^{9} x=\sum_{i} d_{i} x e_{i}$ for all $x \in S$, and therefore ${ }^{g} r=\sum_{i} d_{i} r e_{i}=$ $r \sum_{i} d_{i} e_{i}=r$; so $r \in R$.

3) By the comments above, it is only neccesary to show the second equality. But, by part 2) we have: $Z(R)=R \cap C_{S}(R)=R \cap E=$ $C_{S}(E) \cap E=Z(E)$.

Remark. Note that in proposition 2.3 we showed that if the skew group ring $S * G$ is $H$ separable over the base ring $S$, then the action of $G$ must be $\omega$-outer. Here we obtain the opposite condition, if the ring $S$ is $H$-scparable over the fixed ring $R$, the action of $G$ must be $\omega$ inner. Therefore we cannot have a "chain" of $H$-scparabble extensions in faithful group actions.

Proposition 3.2. Let $S$ be $H$-separable over the fixed ring $R$ and assume there exists a central element in $S$ of trace one. Then $E$ is separable over $Z(S)$ and $H$-separable over $E^{C}$ (so $E$ is an Azumaya algebra).

Proof: The existence of a central element of trace 1 makes the trace map $\operatorname{tr}: S \rightarrow R$ split as a $(R-R)$-bimodule map. Hence $R$ is a direct summand of $S$ as $(R-R)$-bimodules and by [S, proposition 1.3] $E$ is 
separable over $Z(S)$. Furthermore, since $Z(S) \subseteq Z(E)$, the theorem of Azumaya for separable extension over commutative rings implies that $E$ is separable over its center $Z(E)$ and $Z(E)$ is separable over $Z(S)$. Therefore, $E$ is $H$-separable over $Z(E)$, which by proposition 3.1 is equal to the fixed subring $E^{G}$.

The action of $G$ on $S$ induces an action on $E$, but we need to consider the inertia subgroup $K=I(E)$. In this way $G / K$ acts faithfully on $E$. We now describe conditions for $E$ to be a Galois extension of $E^{G}$.

Proposition 3.3. $g \in K$ if and only if $\phi_{g} \subseteq Z(E)$.

Proof: Since $\phi_{g} \subseteq E$ the neccesary condition is obvious. Now let $\alpha \in \phi_{g} \subseteq Z(E)$; then $\alpha\left({ }^{9} x-x\right)=0$ for all $x \in E$ and therefore ${ }^{g} x=x$ for all $x \in E$.

Theorem 3.4. Let $S$ be $H$-separable over $R$ and assume there is a central element of trace $1 . S$ is an $E$-Galois extension of $R$ if and only if $C=E^{G}$ and $K$ is trivial.

Proof: $\Rightarrow$ ) By definition of $E$-Galois extension, $K$ is trivial and the action of $G$ on $E$ is $G$-Galois, morcover by proposition $3.2 E$ is $H$ separable over $E^{G}$. Furthermore, by [S2], $E=\sum_{g} \phi_{g}$ is a direct sum and $\phi_{g}=C x_{g}$, thus proposition 3.3 implies that $Z(E)=C$, so proposition 3.1 gives us the result.

$(\Leftarrow)$ Since $K$ is trivial and the fixed elements in $E$ coincide exactly with the central elements we have that the sum $\sum_{g} \phi_{g}$ is direct; moreover in this case $E=C_{E}\left(E^{G}\right)$ and $E^{G}=Z(E)$ giving us $C_{E}\left(E^{G}\right)$ equal to the direct sum of the correspondent $\phi_{g}^{\prime}$. Thus by [S2, theorem 1.2] the action of $G$ on $E$ is $G$-Galois.

\section{References}

[A] Alfaro R., $T$-Galois Extensions on Rings and a submodule correspondence, Comm. in Algebra, to appear.

[D] DeMeyer F., Some notes in the general Galois Theory of rings, Osaka J. Math. 2 (1965), 117-127.

[DI] DeMeyer F. And Ingraham E., "Separable Algebras over Commutative Rings," Lecture Notes in Mathematics 181, Springer-Verlag, 1971. 
[HI] HiRata K., Some types of separable extension of rings, Nagoya Math. J. 33 (1968), 107-115.

[H2] Hirata K., Separable extensions and centralizers of rings, Nagoya Math. J. 35 (1969), 31-45.

[MS] MCMAHON E. AND SHAPIRO J., On strong and $H$ separability in ordinary and Skew group rings, Houston J. Math. 15, no. 3 (1989).

[MM] MCMahon E. And MEWborn A. C., Separable extensions of non-commutative rings, Hokkaido Math. J. XIII, I (1984).

[OP] Osterburg J. And Peligrad C., A Strong Connes Spectrum for finite group actions of simple rings, J. Algebra, to appear.

[S] Sugano K., On centralizers in separable extensions, Osaka $J$. Math. 7 (1970), 29-40.

[S2] SUGANo K., On a special type of Galois extensions, Hokkaido Math. Journal 9 (1980), 123-128.

[SM] SzETO G. AND MA L., On center-Galois extensions over a ring, Glasnik Matematicki 24 (44) (1989), 11-16.

University of Michigan-Flint

M1 48502

U.S.A.

Rebut ol 25 de Novembre de 1991 
